Active and Passive Elec. Comp., 1989, Vol. 13, pp. 211-227

Reprints available directly from the publisher

Photocopying permitted by license only

(C) 1989 Gordon and Breach Science Publishers, Inc.

Printed in Great Britain

\title{
ANALYTICAL STUDY OF INDUCTOR SIMULATION CIRCUITS
}

\author{
UMESH KUMAR* and SUSHIL KUMAR SHUKLA, AMIETE $\dagger$ \\ *Fellow, IETE; Senior Member, IEEE Department of Electrical Engineering, I.I.T., \\ New Delhi-110016 India \\ †Centre For Development of Telematics, Chanakyapuri, New Delhi-110021 India \\ (Received January 15, 1988; in final form January 31, 1989)
}

\section{INTRODUCTION}

The recent trend in electronics has been toward reducing the size of circuits; a trend which culminated in the development of integrated circuits. Although it is relatively simple to reduce the dimensions of resistors and capacitors it has proven impractical to achieve a comparable reduction in the size of inductors. The main reasons for this are the following [4]:

1. Semiconductors, which provide the building material of integrated circuits, do not exhibit ferromagnetism. Hence, both the magnetic material forming the core and the conductors forming the windings of the inductor must be deposited on the semiconductor surface. This arrangement results in inductors of very low inductance $\mathrm{L}$ and poor quality factor $\mathrm{Q}$.

2. The inherent relation between the physical size of an inductor and its quality factor creates a size problem. If the size of inductor is reduced by replacing every linear dimension 1 by $x 1$, then a new quality factor $Q_{n}$ vill become $x^{2} Q$, where $Q$ is the original one. Thus, reduction in size of inductor reduces the quality factor greatly.

3. Even large inductors are quite lossy. The best attainable $\mathrm{Q}_{\mathrm{L}}$ is about 1,000 ; by contrast, capacitors with $\mathrm{Q}_{\mathrm{C}}$ values of 5,000 to 10,000 can be obtained.

4. For frequencies below $20 \mathrm{~Hz}$ for example, the size and weight of inductors become exceedingly large and $\mathrm{Q}_{\mathrm{L}}$ becomes very low. Hence, inductors are seldom used at such low frequencies.

5. Inductors using ferromagnetic materials are basically nonlinear elements. Hence, unless the amplitude of the signal which they handle 
is kept small and direct currents are avoided, they generate harmonic distortion.

6. Inductors tend to act as small antennas, radiating as well as picking up electromagnetic waves. This can result in undesirable noise and coupling of signals in circuits containing inductors.

Thus, conventional wire-wound inductors are bulky and costly. There also exists fundamental limitations on the realization of inductances for microminiature and integrated circuit applications. As a result, there has been increasing interest in the realization of active RC filters requiring no inductance. The advantages in using active RC filters are the reduction in size, weight, cost, and power consumption, and an increase in the system reliability in comparison to discrete versions.

\section{THEORY}

There have been three distinct techniques of inductance simulation, namely: (i) active $\mathrm{R}$ utilizing the frequency sensitive properties of an Op-Amp; (ii) active RC in which the Op-Amp is modelled by controlled sources with real parameters and resistances; and (iii) techniques based on mechanical resonance in piezoelectric materials.

Active $\mathrm{R}$ methods are usually associated with serious stability and sensitivity problems while the amount of success achieved with the mechanical resonance method appears to be very limited. An active RC circuit is most useful and is widely used for replacing inductances in an LC-resistively terminated network. Such an active RC circuit is expected to have as low a sensitivity as a passive circuit, except for imperfections in the realization of active inductors.

The most commonly used active circuit for realization of the inductor is the gyrator. Symbolically the $\mathrm{Z}$ matrix representation of the gyrator is given by,

$$
\left[\begin{array}{l}
\mathrm{V}_{1} \\
\mathrm{~V}_{2}
\end{array}\right]=\left[\begin{array}{cc}
\mathrm{O} & -\mathrm{K} \\
\mathrm{K} & \mathrm{O}
\end{array}\right]\left[\begin{array}{l}
\mathrm{I}_{1} \\
\mathrm{I}_{2}
\end{array}\right]
$$

where $\mathrm{K}$ is known as the gyration resistance. In the circuit in Fig. 1, the input impedance seen at port $1-1^{\prime}$, if port $2-2^{\prime}$ is terminated in a capacitor $C$, is $Z_{\text {in }}=K^{2} S C$. This corresponds to an inductor of value $K^{2} C$ henries. 

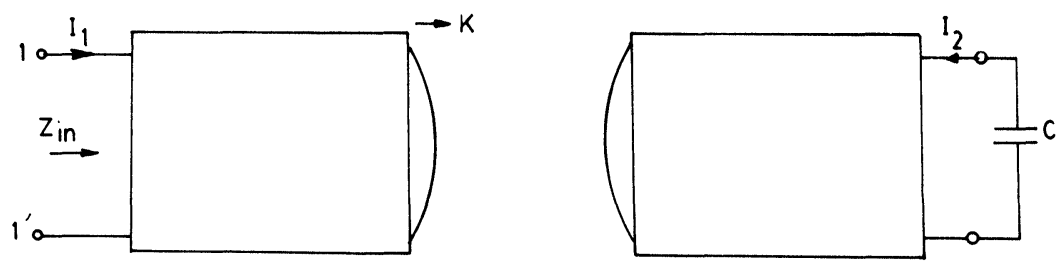

FIGURE 1

Therefore, the gyrator, terminated in a capacitor, can be used to realize an inductor.

The gyrator can be realized by using Riordan's circuit shown in Fig. 2 . In this diagram, the gain of the Op-Amp is assumed to be infinite, then the circuit will behave as a gyrator with terminals $1-1^{\prime}$ and $2-2^{\prime}$. Input impedance at 1-1' can be given by

$Z_{\text {in }}=\frac{R_{1} R_{3} R_{5}}{R_{4}} \cdot Z_{2}$

If $\mathrm{Z}_{2}$ is the impedance at terminals $2-2^{\prime}$.

\section{TYPICAL CIRCUITS}

We can divide all the circuits into three categories:

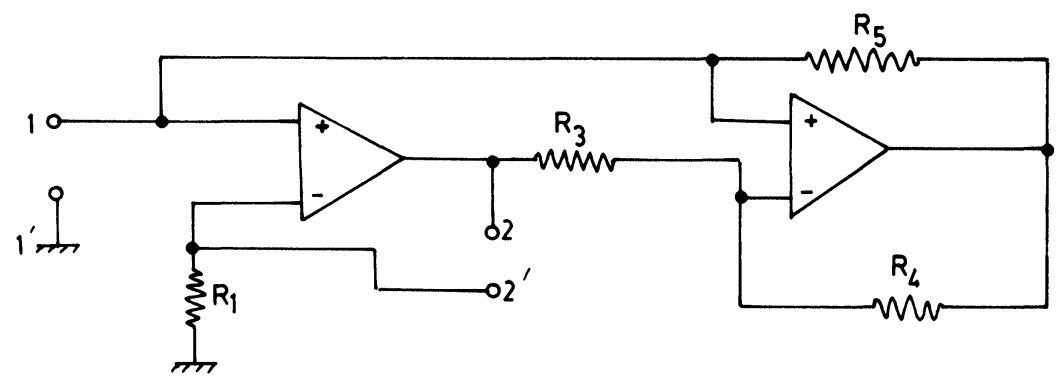

FIGURE 2 


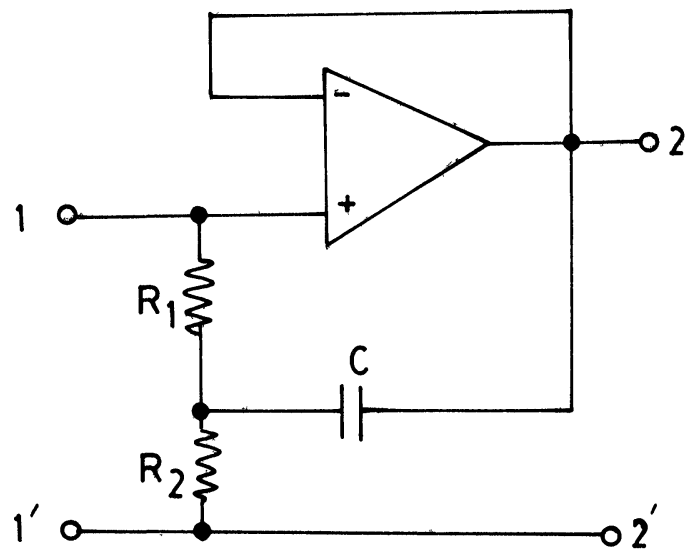

FIGURE 3

\section{A. Single Op-Amp Grounded Inductance Realization}

There are two such circuits. The circuit diagrams and input-impedances are shown here in Figs. 3 and 4.

$$
\begin{aligned}
\mathrm{Z}_{\text {in }}=\mathrm{R}_{1}+\mathrm{R}_{2}+\mathrm{SR}_{1} \mathrm{R}_{2} \mathrm{C} \quad \mathrm{L} & =\frac{\mathrm{R}_{2} \mathrm{C}\left(\mathrm{R}_{1}-\mathrm{R}_{2}\right)}{1+\mathrm{w}^{2} \mathrm{R}_{2}^{2} \mathrm{C}^{2}} \\
\mathrm{Q} & =\frac{\mathrm{wC}\left(\mathrm{R}_{1}-\mathrm{R}_{2}\right)}{1+\mathrm{w}^{2} \mathrm{C}^{2} \mathrm{R}_{2}^{2}}
\end{aligned}
$$

The circuit of Fig. 3 realizes a lossy inductor of inductance $L=R_{1} R_{2} C$ and $R=R_{1}+R_{2}$. The quality factor of this circuit is poor.

Circuit of Fig. 4 realizes an inductor that is a function of frequency and is not suitable for wide range of frequency.

\section{B. Grounded Inductor Simulation Using Two Op-Amp}

The two commonly used circuits are Riordan's and Antoniou's circuits. The Riordan's circuit is shown in Fig. 2, with capacitor $C_{2}$ across 2-2'. The Antoniou's circuit is shown in Fig. 5. For both circuits 


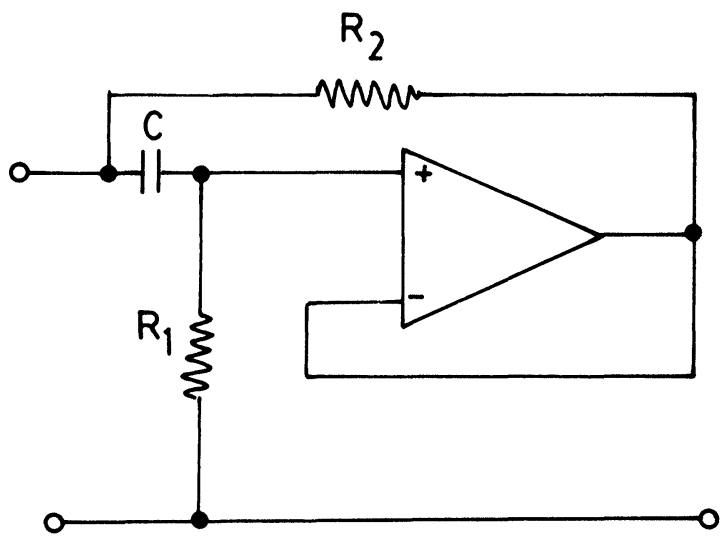

FIGURE 4

$$
\mathrm{Z}_{\text {in }}=\frac{\mathrm{R}_{1} \mathrm{R}_{3} \mathrm{R}_{5}}{\mathrm{R}_{4}} \mathrm{SC}_{1}
$$

or $\mathrm{Z}_{\text {in }}=\mathrm{SR}^{2} \mathrm{C} \quad$ with $\mathrm{R}_{1}=\mathrm{R}_{3}=\mathrm{R}_{4}=\mathrm{R}_{5}=\mathrm{R}$

Input impedance $Z_{\text {in }}$ is equivalent to an ideal inductance, and its value is given by $\mathrm{L}=\mathrm{R}^{2} \mathrm{C}$ henry.

These two circuits are equivalent to the realization of an active RC inductance with a gyration resistance $K=R$. Since one of the input terminals is grounded, the circuit can realize only grounded inductors.

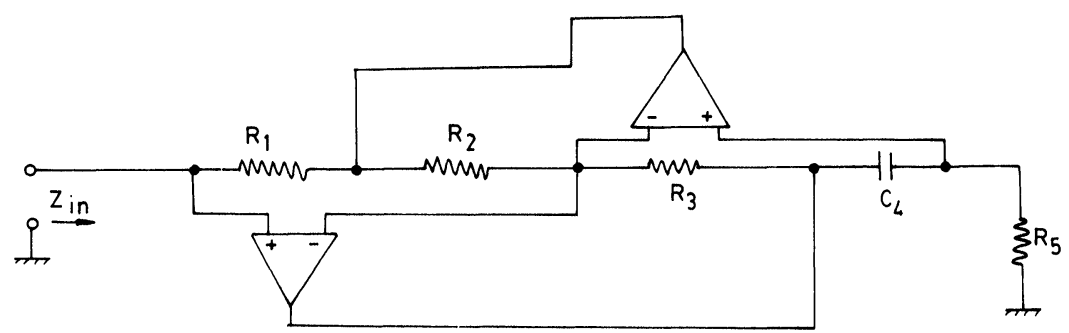

FIGURE 5 


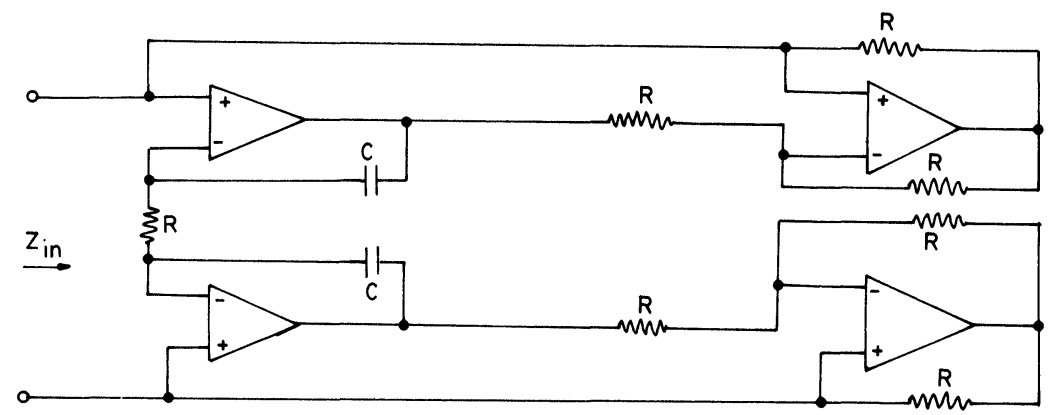

FIGURE 6

\section{Realisation of Floating Inductance}

The realization of floating inductance requires two gyrators connected backto-back as shown in Fig. 6. An analysis of this circuit will show that the input impedance at port $1-1^{\prime}$ is equal to $\mathrm{SR}^{2} \mathrm{C}$; that is, an inductance valued $\mathrm{R}^{2} \mathrm{C}$.

We have seen that in each circuit, inductance can be given by $L=C^{2}$ and we can choose the suitable value of $C$ and $R$ for given values of $L$. Suggested values for $C$ are in the range of $100 \mu F$ to $0.01 \mu F$. The corresponding value of the $R$ can be calculated from the expression for $L$.

\section{Experiments}

The chosen values of $R$ and $C$ were $4.7 \mathrm{~K} \Omega$ and $0.1 \mu \mathrm{F}$ respectively. Thus, simulated inductance was

$\mathrm{L}=4.7^{2} \times 0.1=2.209 \mathrm{H}$

Simulated inductance was then measured by an L-C-R bridge and by L-C circuits illustrated in Fig. 7 and Fig. 8. For grounded inductance simulation, the circuit in Fig. 7 was used, and for floating inductor, the circuit in Fig. 8 was used.

The simulation of inductor was done using Riordan (Fig. 2) and Antonious (Fig. 5) circuits and the circuit in Fig. 6. A 741 Op-Amp was used.

The grounded inductors were measured by the circuit in Fig. 7 and the floating inductor by the circuit in Fig. 8. 


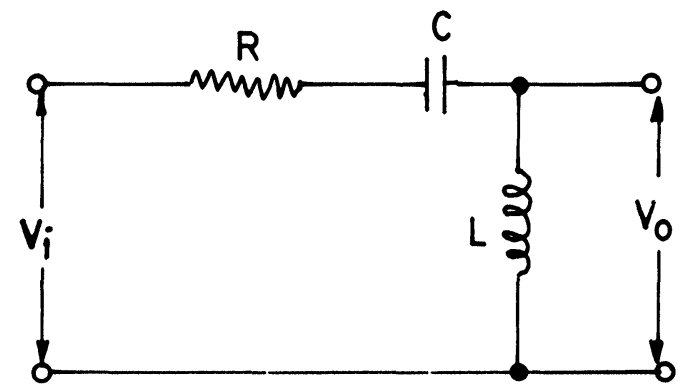

FIGURE 7

The input $V_{i}$ and output $V_{o}$ voltages of the circuits (Fig. 6) and (Fig. 7) were measured with a cathode ray oscilloscope; the phase difference between them was also measured. Input $V_{i}$ was given from an oscillator having a range up to about $1 \mathrm{MHz}$. Input and output voltages were measured at various frequencies and then calculations for inductances were performed.

\section{Calculations}

For the circuit in Fig. 7

$$
V_{o}=\frac{S L V_{i}}{R+S L+1 / C S} \text {, where } R \text { is the small series resistance. }
$$

or

$\frac{V_{o}}{V_{i}}=\frac{S L}{R+S L+1 / C S}=\frac{S^{2} L C}{L C S^{2}+R C S+1}$

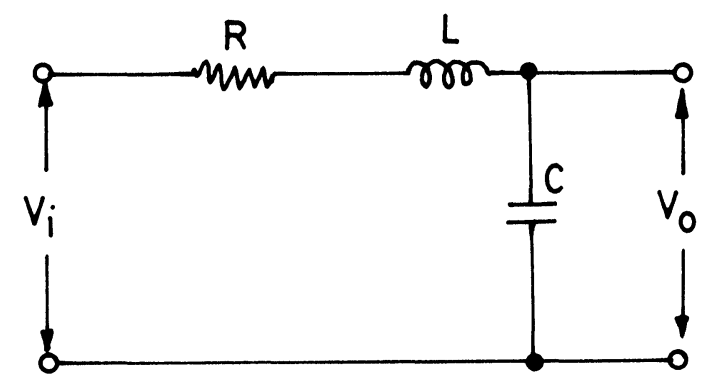

FIGURE 8 
Let
$\left|\frac{\mathrm{V}_{\mathrm{o}}}{\mathrm{V}_{\mathrm{i}}}\right|=\mathrm{A}_{1}$ and $/ \frac{\mathrm{V}_{\mathrm{o}}}{\mathrm{V}_{\mathrm{i}}}=\phi_{1}$

Then

$$
\begin{aligned}
\frac{V_{o}}{V_{i}}(j w) & =\frac{-w^{2} L C}{-w^{2} L C+j w R C+1} \\
\therefore A_{1} & =\frac{w^{2} L C}{\sqrt{\left(1-w^{2} L C\right)^{2}+w^{2} R^{2} C^{2}}}
\end{aligned}
$$

and $\phi=-\tan ^{-1} \frac{w R C}{w^{2} L C-1}$ or $\left(w^{2} L C-1\right) \tan \phi=-w R C$

Substituting the value of $\mathrm{wRC}$ in the above equation for $\mathrm{A}_{1}$

$$
\begin{aligned}
& A_{1}^{2}=\frac{\left(w^{2} L C\right)^{2}}{\left(1-w^{2} L C\right)^{2}+\left(1-w^{2} L C\right)^{2} \tan ^{2} \phi} \\
& \text { or } A_{1}^{2}=\frac{\left(w^{2} L C\right)^{2}}{\left(1-w^{2} L C\right)^{2} \operatorname{Sec}^{2} \phi_{1}} \text { or } A_{1}= \pm \frac{w^{2} L C}{\left(1-w^{2} L C\right) \operatorname{Sec} \phi_{1}} \\
& \text { or } \frac{w^{2} L C}{1-w^{2} L C}= \pm \frac{A_{1}}{\operatorname{Cos} \phi_{1}} \\
& \text { or } \\
& \frac{2 w^{2} L C-1}{1}=\frac{A_{1}-\operatorname{Cos} \phi_{1}}{A_{1}+\operatorname{Cos} \phi_{1}} \text { or } \frac{A_{1}+\operatorname{Cos} \phi_{1}}{A_{1}-\operatorname{Cos} \phi_{1}}
\end{aligned}
$$

or

$$
\begin{aligned}
& 2 w^{2} L C=1+\frac{A_{1}-\operatorname{Cos} \phi_{1}}{A_{1}+\operatorname{Cos} \phi_{1}} \quad \text { or } \quad 1+\frac{A_{1}+\operatorname{Cos} \phi_{1}}{A_{1}-\operatorname{Cos} \phi_{1}} \\
& =\frac{2 A_{1}}{A_{1}+\operatorname{Cos} \phi_{1}} \quad \text { or } \frac{2 A_{1}}{A_{1}-\operatorname{Cos} \phi_{1}} \\
& \therefore \quad L=\frac{1}{w^{2} C}\left[\frac{A_{1}}{A_{1}+\operatorname{Cos} \phi_{1}}\right] \text { or } \frac{1}{w^{2} C} \frac{A_{1}}{\left(A_{1}-\operatorname{Cos} \phi_{1}\right)} \\
& \text { or } \mathrm{L}=\frac{1}{\mathrm{w}^{2} \mathrm{C}} \cdot \frac{\mathrm{A}_{1}}{\mathrm{~A}_{1} \pm \operatorname{Cos} \phi_{1}}
\end{aligned}
$$




$$
\begin{aligned}
\therefore \quad \mathrm{wRC}=-\left(\mathrm{w}^{2} \mathrm{LC}-1\right) \tan \phi_{1} & =-\left(\frac{\mathrm{A}_{1}}{\mathrm{~A}_{1} \pm \operatorname{Cos} \phi_{1}}-1\right) \tan \phi_{1} \\
& =\left(1-\frac{\mathrm{A}_{1}}{\mathrm{~A}_{1} \pm \operatorname{Cos} \phi_{1}}\right) \tan \phi_{1} \\
& =\left(\frac{ \pm \operatorname{Cos} \phi_{1}}{\mathrm{~A}_{1} \pm \operatorname{Cos} \phi_{1}}\right) \tan \phi_{1}
\end{aligned}
$$

Since $\phi$ is always positive in this case;

$$
\begin{aligned}
& =\frac{ \pm \operatorname{Sin} \phi_{1}}{\mathrm{~A}_{1} \pm \operatorname{Cos} \phi_{1}} \\
& \mathrm{R}=\frac{1}{\mathrm{wC}} \frac{\operatorname{Sin} \phi_{1}}{\mathrm{~A}_{1}+\operatorname{Cos} \phi_{1}} \therefore \mathrm{L}=\frac{1}{\mathrm{w}^{2} \mathrm{C}} \frac{\mathrm{A}_{1}}{\mathrm{~A}_{1}+\operatorname{Cos} \phi_{1}}
\end{aligned}
$$

So, with the circuit in Fig. 7:

$$
\mathrm{R}=\frac{1}{\mathrm{wC}} \frac{\operatorname{Sin} \phi_{1}}{\mathrm{~A}_{1}+\operatorname{Cos} \phi_{1}} \quad \text { and } \quad \mathrm{L}=\frac{1}{\mathrm{wC}} \frac{\mathrm{A}_{1}}{\mathrm{~A}_{1}+\operatorname{Cos} \phi_{1}}
$$

When the circuit in Fig. 8 is considered,

$$
\frac{V_{o}}{V_{i}}=\frac{1 / C S}{R+S L+1 / C S}=\frac{1}{L_{C S}+R C S+1}
$$

or

$$
\begin{aligned}
& A_{2} \phi_{2}=\frac{1}{-L C w^{2}+j w C R+1} \\
& \therefore A_{2}^{2}=\frac{1}{\left(1-w^{2} L C\right)^{2}+w^{2} C^{2} R^{2}} \quad \text { and } \tan \phi_{2}=-\frac{w C R}{1-L C w^{2}} \\
& \therefore \quad A_{2}^{2}=\frac{1}{\left(1-w^{2} L C\right)^{2} \operatorname{Sec}^{2} \phi_{2}} \text { or } \quad 1-w^{2} L C= \pm \frac{\operatorname{Cos} \phi_{2}}{A_{2}} \\
& \text { or } L=\frac{1}{w^{2} C}\left[1 \mp \frac{\operatorname{Cos} \phi_{2}}{A_{2}}\right] \\
& \text { Now, } w C R=\left(w^{2} L C-1\right) \tan \phi_{2} L C=1 \mp \frac{\operatorname{Cos} \phi_{2}}{A_{2}}
\end{aligned}
$$


$=\mp \frac{\operatorname{Cos} \phi_{2}}{A_{2}} \tan \phi_{2}=\mp \frac{\operatorname{Sin} \phi_{2}}{A_{2}}$

For this circuit, either output voltage is always lagging or $\phi_{2}$ is negative. Thus, the lower sign should be considered.

$\mathrm{R}=\frac{\operatorname{Sin} \phi_{2}}{\mathrm{wC} \mathrm{A}_{2}} \quad$ and $\quad L=\frac{1}{\mathrm{w}^{2} \mathrm{C}}\left[1+\frac{\operatorname{Cos} \phi_{2}}{\mathrm{~A}_{2}}\right]$

Based on the above two derivations, calculations were performed for inductance at various frequencies and results are plotted in Fig. 9. In the ideal case, $\mathrm{Q}$ of the circuit becomes infinite, but experimentally we find some finite equivalent resistance and hence finite $Q$.

As the value of inductance is increased, the simulated inductance starts decreasing at higher frequency. The result has also been verified by computer-based analytical calculation. With $\mathrm{L}=22.09 \mathrm{H}$, the simulated inductance starts falling at frequency of $200 \mathrm{kHz}$ for Antoniou and at about $500 \mathrm{kHz}$ for Riordan's circuit. For $\mathrm{L}=2.209 \mathrm{H}$, corresponding frequencies are $2 \mathrm{MHz}$ and $10 \mathrm{MHz}$ respectively.

\section{Analysis of Circuits and Explanation of Graph}

We see that simulated inductance drops to a very low value as frequency is increased. This can be interpreted by considering the practical Op-Amp 741 as non-ideal and with a finite gain A. With the help of following derivation for Riordan's circuit, the variation of inductance with frequency can be obtained.

The various currents in Riordan's circuit are shown in Fig. 10. The gain of the Op-Amp is assumed finite and equal to A. Equations at various nodes can be written as:

$$
\begin{aligned}
& \left(v-v_{1}\right) A=v_{3} \\
& \left(v-v_{2}\right) A=v_{4} \\
& v_{4}=v-i R=v_{2}-i_{1 R} \\
& v_{4}=v_{2}+i_{1 R} \\
& v_{3}=v_{1}+\frac{v_{1}}{S C R}
\end{aligned}
$$




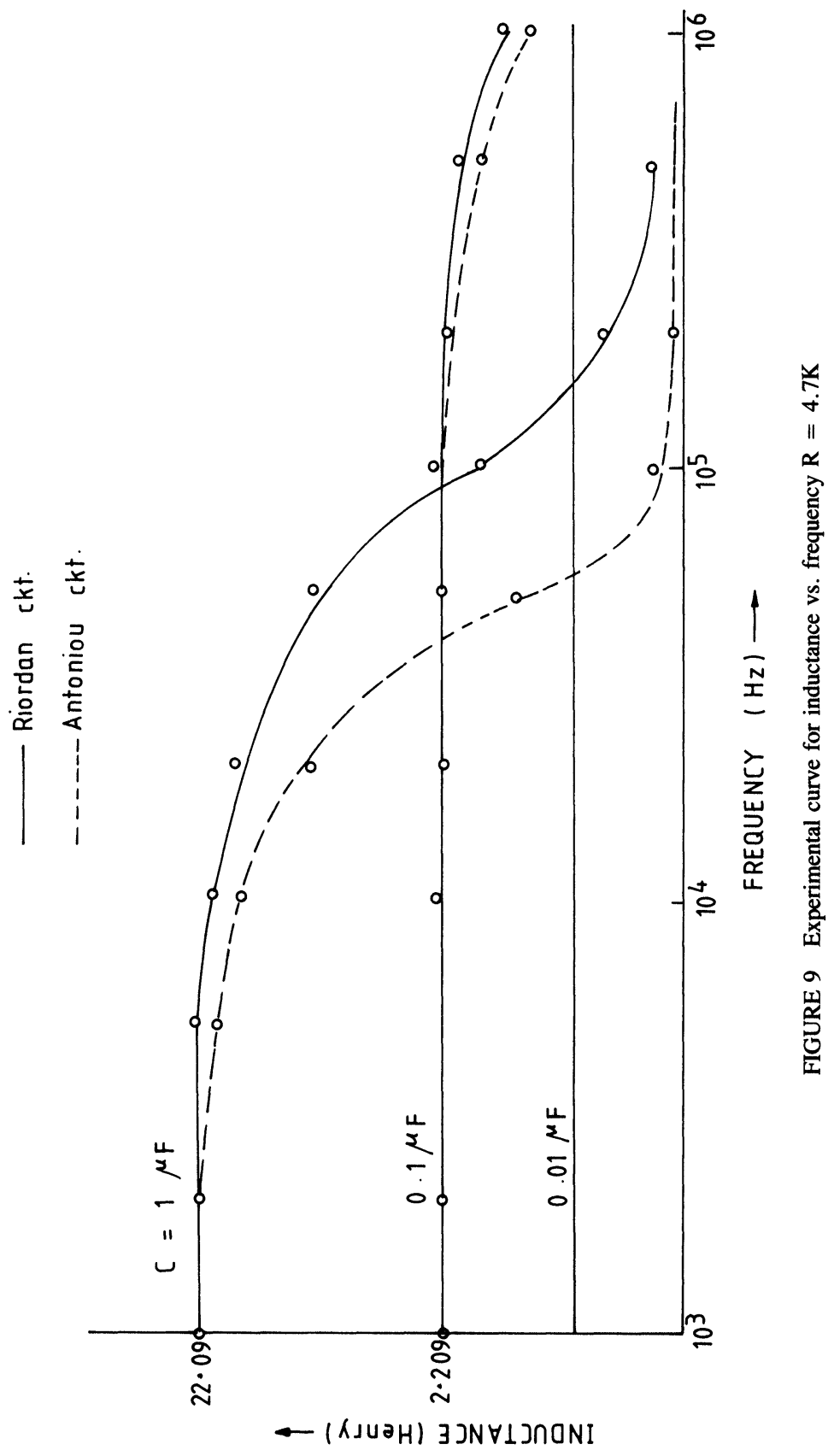




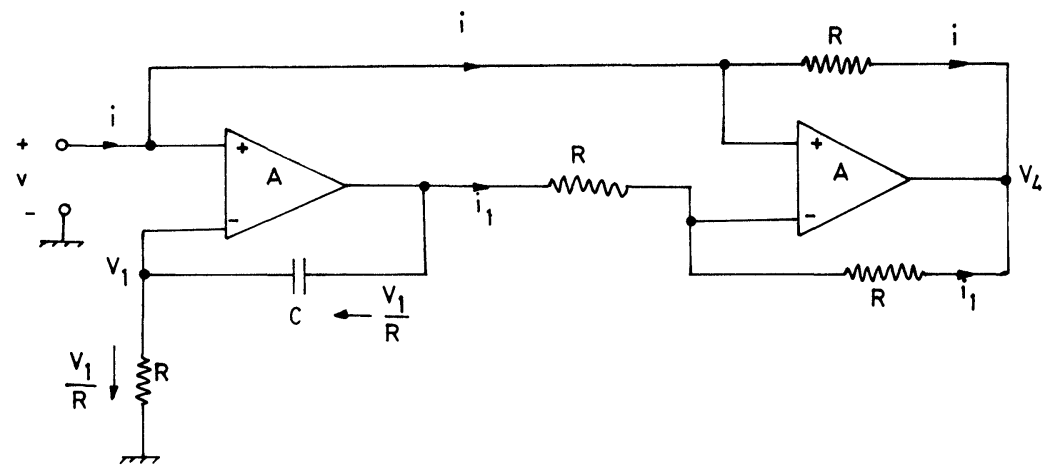

FIGURE 10

On solving these equations and finding input impedance $Z_{\text {in }}$ we find:

$Z_{\text {in }}=\frac{v}{i}=\frac{C R^{2} S+\frac{R}{1+A}}{\frac{C R S}{1+A}+\frac{A-1}{A+3}}$

Substituting $S=j w$, and rationalizing, we have,

$Z_{\text {in }}=R_{e q}+j w L_{e q}$

where $\quad L_{\text {eq }}=\frac{C^{2}\left[\frac{A-1}{A+3}-\frac{1}{(A+1)^{2}}\right]}{\left(\frac{A-1}{A+3}\right)^{2}+\frac{w^{2} R^{2} C^{2}}{(1+A)^{2}}}$

and $\quad R_{\text {eq }}=\frac{\left[R \frac{A-1}{A(A+4)}+\frac{w^{2} C^{2} R^{2}}{1+A}\right]}{\left(\frac{A-1}{A+3}\right)^{2}+\frac{w^{2} R^{2} C^{2}}{(1+A)^{2}}}$

It can be seen from the above expression that $\mathrm{L}_{\mathrm{eq}}$ is inversely proportional to $w^{2}$, and as $w$ increases, $L_{e q}$ approaches zero. The values of $L_{e q}$ at various $\mathrm{w}$ are also calculated by computer and these are shown in Fig. 12 . 


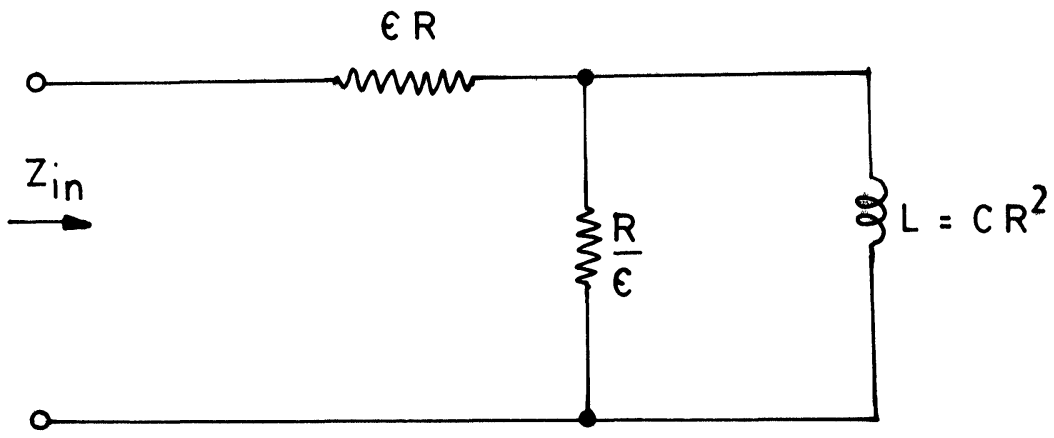

FIGURE 11

Similar analysis for Antoniou's circuit gives:

$L_{e q}=\frac{C R^{2}\left(A^{2}+2 A-3\right)}{(A+1)^{2}+4 w^{2} C^{2} R^{2}}$

$$
\mathrm{Z}=\frac{\mathrm{R}\left[\mathrm{SCRA}^{2}+2(1+\mathrm{SCR})(1+\mathrm{A})\right]}{\mathrm{A}^{2}+2(1+\mathrm{SCR})(1+\mathrm{A})}
$$

$R_{e q}=\frac{R(1+A)\left(2+4 w^{2} C^{2} R^{2}\right)}{(A+1)^{2}+4 w^{2} C^{2} R^{2}}$

These are also calculated and are plotted in Fig. 13.

Any discrepancy between experimental and theoretical calculation of variation of inductance with frequency is basically due to non-ideality of the Op-Amp, while theoretical calculations would be more accurate if we replace gain $A$ by $A_{o} / S+w_{o}$.

Another reason for the faster decrease of inductance L may be the existence of a finite resistance ER in the open circuited gyrator. The equivalent input impedance $Z_{\text {in }}$ will be as shown in Fig. 11. Here,

$$
\begin{aligned}
Z_{\text {in }} & =E R+\frac{\frac{R}{E} S L}{\frac{R}{E}+S L} \\
& =E R+\frac{R L S}{R+E L S}
\end{aligned}
$$




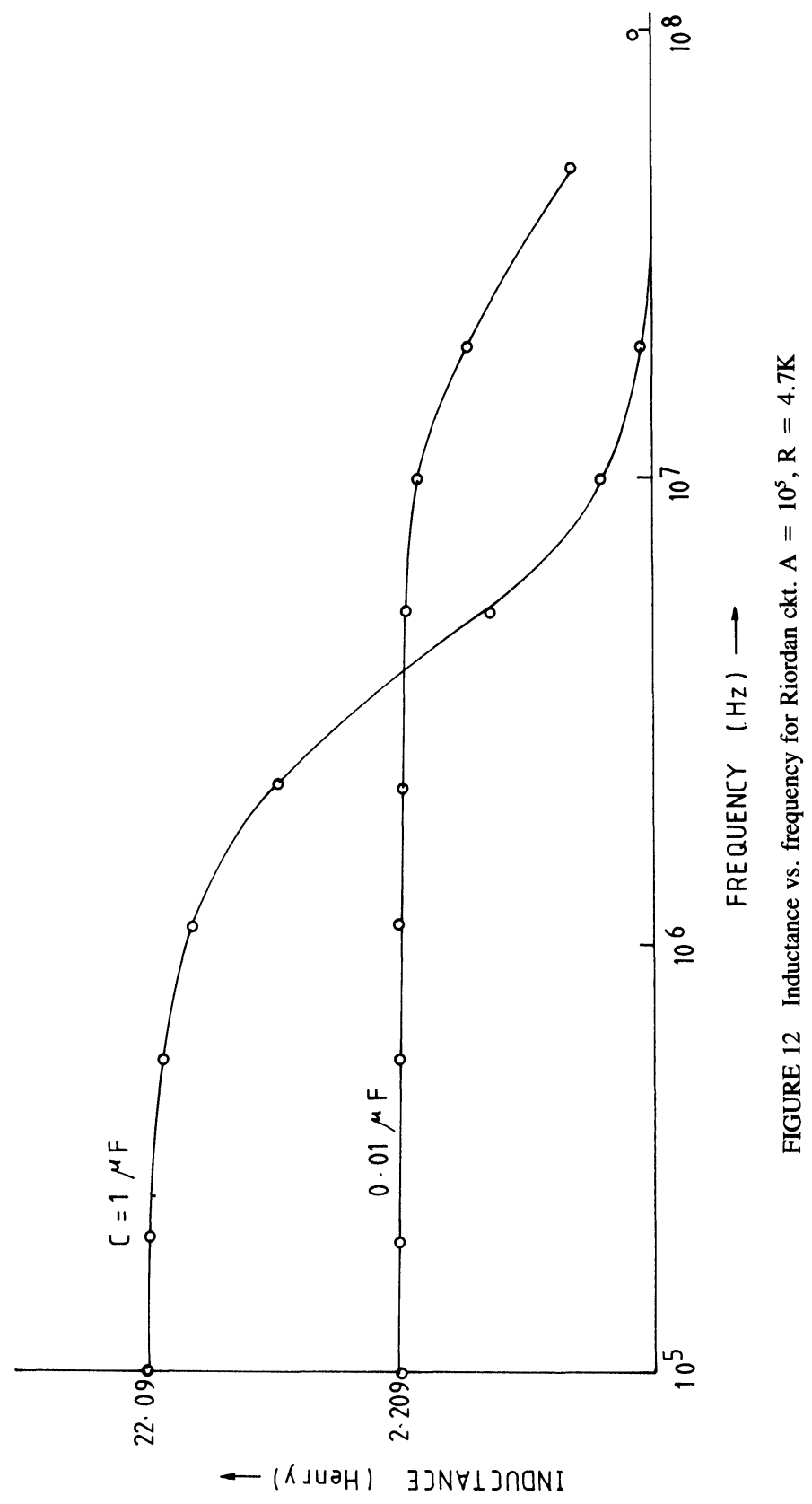




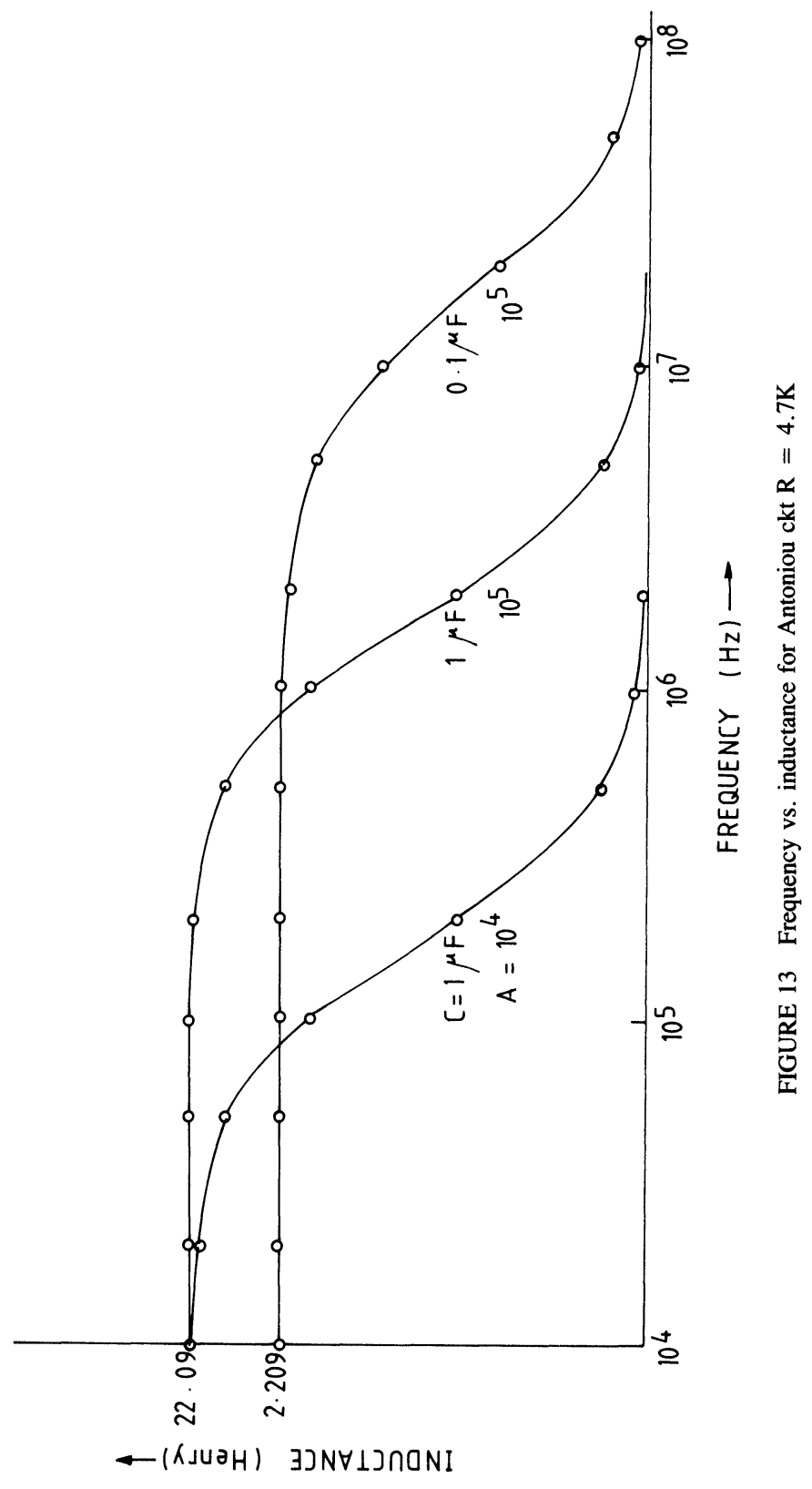


With $S=j w$, and rationalizing,

$$
Z_{\text {in }}=E R+\frac{R L S(R-E L S)}{R^{2}-E^{2} L^{2} S^{2}}=R+\frac{-S^{2} L^{2} R E+S R^{2} L}{\left(R^{2}-E^{2} L^{2} S^{2}\right)}
$$

or $\quad Z_{\text {in }}=E R+\frac{L^{2} R w^{2}+j w L R^{2}}{R^{2}+E^{2} L^{2} w^{2}}$

Thus, $\mathrm{L}_{\mathrm{eq}}=\frac{\mathrm{LR}^{2}}{\mathrm{R}^{2}+\mathrm{E}^{2} \mathrm{~L}^{2} \mathrm{w}^{2}}$

For low frequencies, the effect of ER is negligible as $0<\mathrm{E} \ll 1$, but as frequency increases, the equivalent inductance $\mathrm{L}_{\mathrm{eq}}$ decreases.

\section{CONCLUSIONS}

Inductors using ferromagnetic materials are bulky and costly, but a reduction in the size of the inductor reduces the quality factor. There also exist fundamental limitations on the realization of inductances for microminiature and integrated circuit applications. As a result, RC-active devices are used to simulate inductors on silicon chips.

In this study, the realization of inductors using three basic circuits (a) Riordan's circuit, (b) Antoniou's circuit, and (c) Floating inductor circuit was studied. Inductance of the simulated circuit was measured experimentally at various frequencies. The circuits were analysed using nonideal active devices and their performance calculated.

The value of inductance decreases with increasing frequency. This was determined both experimentally as well as by a mathematical model based on finite gain. However, the inductance was observed to decrease at lower frequencies.

The reasons for this lower frequency drop may be (i) finite gyrator resistance ER, and (ii) the gain of the active device is not only finite, but varies with frequency. The effect of ER is shown analytically. The effect of variation of gain with frequency can be shown by replacing the active device by its single pole model.

\section{REFERENCES}

1. G.C. Temes and J.W. Lapatra, "Introduction to Circuit Synthesis and Design," McGraw Hill, 1977. 
2. Dutta Roy, S.C., "A High Q Inductance Transistor Ckt and a tuned oscillator for Microminiature applications", Int. J. Electronics 1963, 18, pp. 1-16.

3. Dutta Roy, S.C., "Operational Amplifier Simulation of a Grounded Inductance: General characteristics and a critical comparison of various circuits", AEÜ, March 1975.

4. R. Cuppens, H.J. De Man and W.M.C. Sansen, "Simulation of large on-chip capacitors and inductors", IEEE J. solid state circuits, vol. SC-14, no. 3, pp. 543-547, June 1979. 

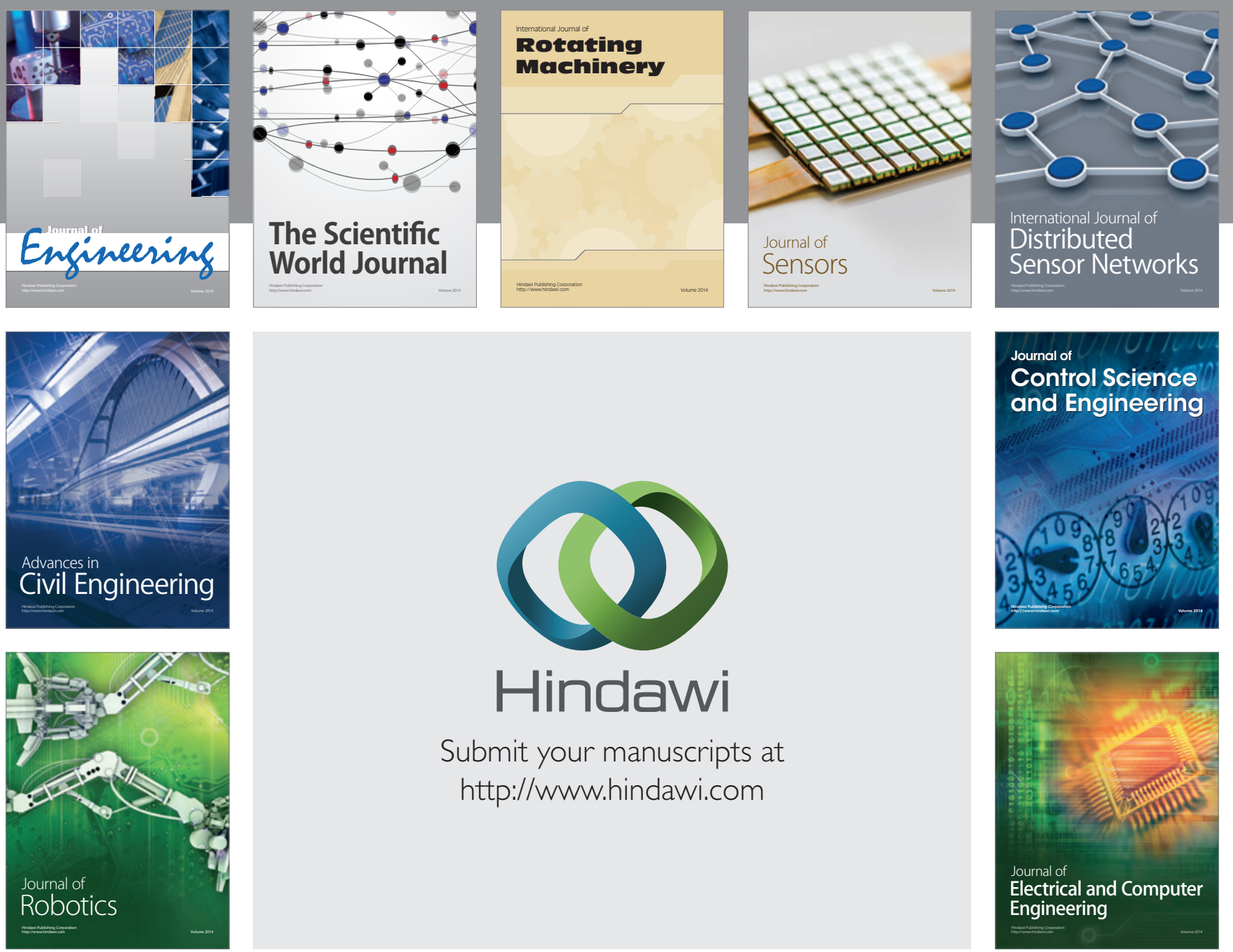

Submit your manuscripts at

http://www.hindawi.com
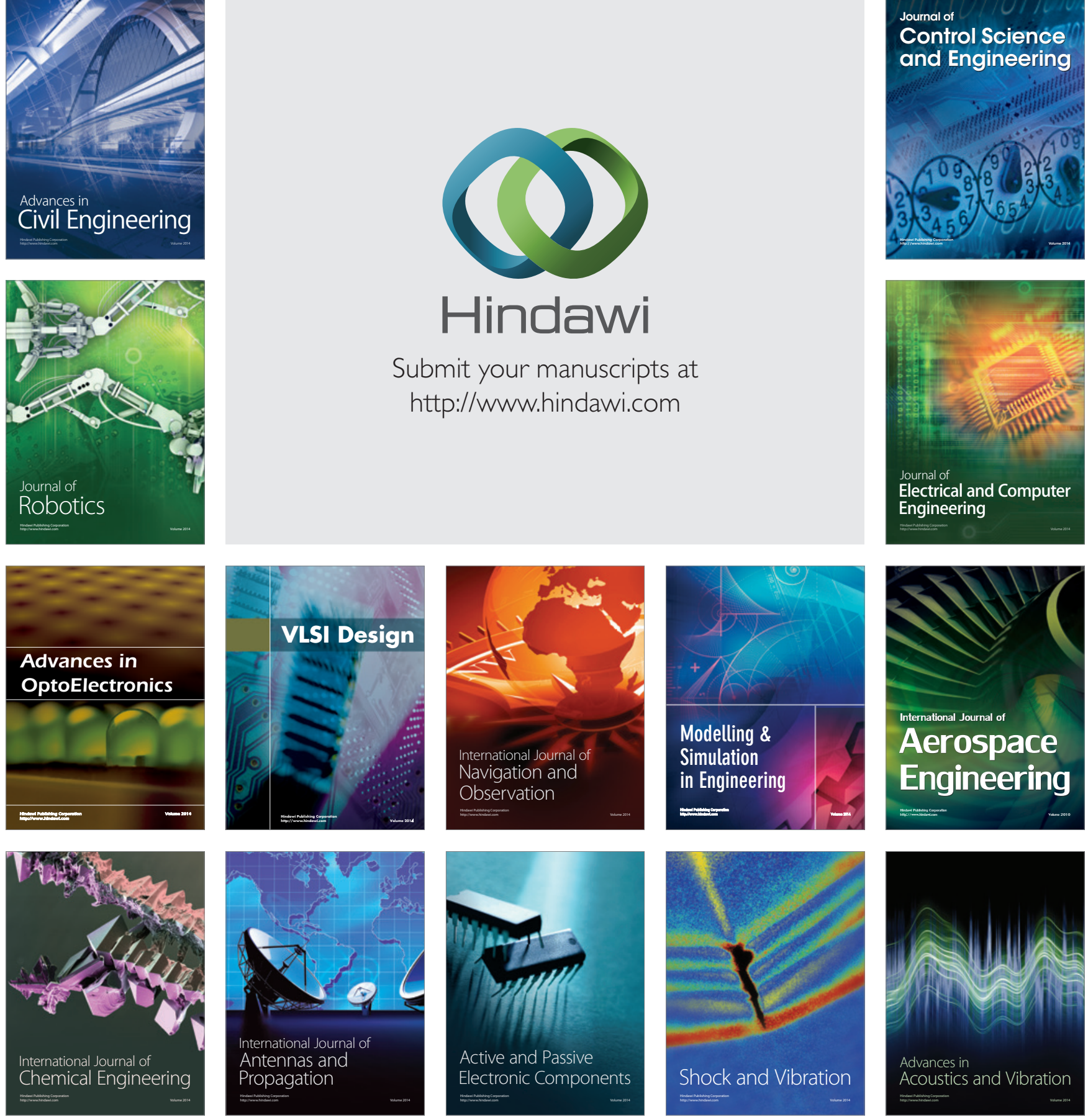